\title{
Improvement in HOMA-IR is an independent predictor of reduced carotid intima-media thickness in obese adolescents participating in an interdisciplinary weight-loss program
}

\author{
Priscila de Lima Sanches ${ }^{1}$, Marco Túlio de Mello ${ }^{1,2,3}$, Natália Elias ${ }^{3}$, Francisco Antonio Helfenstein Fonseca ${ }^{4}$, \\ Aline de Piano ${ }^{1}$, June Carnier ${ }^{1}$, Lila Missae Oyama ${ }^{1,5}$, Lian Tock ${ }^{1}$, Sergio Tufik ${ }^{2,3}$ and Ana Raimunda Dâmaso ${ }^{1,6}$
}

The aim of this study was to verify whether a 1-year interdisciplinary weight-loss program improved common carotid artery intima-media thickness (IMT) and whether insulin resistance and/or inflammation (as measured by the markers plasminogen activator inhibitor type-1 and adiponectin) might underlie obesity in adolescents. A group of 29 post-pubescent obese adolescents were submitted to an interdisciplinary intervention over the course of 1 year. Common carotid artery IMT was determined ultrasonographically. Body composition, blood pressure (BP), glycemia, insulinemia, homeostasis model assessment of insulin resistance (HOMA-IR), lipid profile and adipokine concentrations were analyzed before and after the intervention. The interdisciplinary weight-loss program promoted a significant improvement in body composition, insulin concentration, HOMA-IR, lipid profile, BP and inflammatory state, in addition to significantly decreasing the common carotid artery IMT. Furthermore, this study demonstrated that the difference between baseline and final values of HOMA-IR ( $\triangle$ HOMA-IR) was negatively correlated with concomitant changes in the adiponectin concentration ( $\Delta$ adiponectin; $r=-0.42 ; P=0.02$ ) and positively correlated with changes in common carotid artery IMT ( $\Delta$ carotid IMT; $r=0.41 ; P=0.03$ ). Multiple regression analysis adjusted by age, cardiovascular risk factors and inflammatory markers showed that $\triangle$ HOMA-IR was an independent predictor of significant changes in common carotid artery IMT. This investigation demonstrated that an interdisciplinary weight-loss program promoted a reduction of the common carotid artery IMT in obese Brazilian adolescents, and the improvement of HOMA-IR was an independent predictor of carotid IMT changes in this population.

Hypertension Research (2011) 34, 232-238; doi:10.1038/hr.2010.225; published online 2 December 2010

Keywords: adiponectin; atherosclerosis; inflammation; obesity; PAI-1

\section{INTRODUCTION}

The prevalence of childhood obesity in Brazil has almost tripled over the past 30 years, and $\sim 23 \%$ of Latin American children and adolescents are overweight. ${ }^{1,2}$ The relationship between obesity in childhood and adolescence, and the development of hemodynamic, thrombotic and inflammatory disorders, such as atherosclerosis, later in life is well supported in the literature. ${ }^{3}$

Atherosclerosis can begin in relatively young individuals and continue to develop for many decades before clinical signs are observed or cardiovascular events occur. The first subclinical sign of atherosclerosis is an increase in arterial intima-media thickness (IMT), the result of endothelial dysfunction that leaves lipid deposits in the intima of systemic arteries. ${ }^{4}$ Many hypotheses link obesity, endothelial dysfunction and subclinical signs of atherosclerosis in obese subjects. The majority of research shows that the increase in IMT in obese individuals is influenced by plasma lipid concentration, hypertension, inflammatory state, physical inactivity and impaired glucose metabolism. ${ }^{5-7}$

Among these precursors, insulin resistance seems to be a strong risk factor for the development of atherosclerosis. The negative correlation between insulin resistance and adiponectin may serve as a link between obesity and atherosclerosis. Adiponectin is an anti-inflammatory adipokine that has an insulin-like role in promoting vasodilatation and anti-atherosclerotic effects, stimulating nitric oxide production in endothelial cells and inhibiting the release of proinflammatory adipokines, which in turn contribute to a coupling of vascular insulin

\footnotetext{
${ }^{1}$ Post-Graduate Program of Nutrition, Universidade Federal de São Paulo, São Paulo, Brazil; ${ }^{2}$ Psychobiology Department, Universidade Federal de São Paulo, São Paulo, Brazil; ${ }^{3}$ Research Funding of Psychopharmacology, Universidade Federal de São Paulo, São Paulo, Brazil; ${ }^{4}$ Cardiology Division, Department of Medicine, Universidade Federal de São Paulo, São Paulo, Brazil; ${ }^{5}$ Physiology Department, Universidade Federal de São Paulo, São Paulo, Brazil and ${ }^{6}$ Biosciences Department, Universidade Federal de São Paulo, São Paulo, Brazil

Correspondence: Professor AR Dâmaso or PL Sanches, CEPE/GEO, Rua Francisco de Castro, 93 — Vila Clementino, São Paulo 04020-050, Brazil.

E-mail: ana.damaso@unifesp.br and priscila.sanches@unifesp.br
}

Received 16 June 2010; revised 3 August 2010; accepted 11 August 2010; published online 2 December 2010 
resistance and endothelial dysfunction. ${ }^{8,9}$ Moreover, a decrease in insulin sensitivity is related to increases in the endothelial expression of plasminogen activator inhibitor type-1 (PAI-1) and cellular/vascular adhesion molecules implicated in the initiation of endothelial dysfunction. ${ }^{4,9,10}$

The prevalence of cardiovascular diseases in children and adolescents is increasing, and there is evidence that carotid IMT is higher in children with diabetes, hypercholesterolemia and obesity than in healthy controls. ${ }^{4,5,6,11}$ Some studies of the effects of exercise training, either associated with dietary changes or alone, showed that weight loss and physical exercise decrease insulin resistance and improve endothelial dysfunction in adults. ${ }^{12,13}$ However, few studies have investigated the effects of 1 year of interdisciplinary intervention in this parameter in obese adolescents. ${ }^{14,15}$

The aim of this investigation was to verify whether 1 year of an interdisciplinary weight-loss program improved common carotid artery IMT and whether insulin resistance and/or inflammation (as measured by the levels of markers, plasminogen activator inhibitor type-1 and adiponectin) might underlie obesity in Brazilian adolescents.

\section{METHODS}

\section{Study subjects}

A total of 29 post-pubescent ${ }^{16}$ obese adolescents (body mass index (BMI) $>95$ th percentile on the Centers for Disease Control and Prevention (CDC) reference growth charts), ${ }^{17}$ aged from 14 to 19 years $(16.03 \pm 1.59$ years), including 17 girls and 12 boys, were recruited for an interdisciplinary weight-loss program that lasted for 1 year. The inclusion criteria for the postpubertal stage were based on the Tanner scale (stage five) for both boys and girls. Non-inclusion criteria were as follows: other metabolic or endocrine diseases; chronic alcohol consumption; previous use of drugs, such as anabolicandrogenic steroids or psychotropics, which may affect appetite regulation; and pregnancy. Informed parental consent and the adolescents' assent to participate as volunteers in an interdisciplinary weight-loss program were obtained. This study was conducted in accordance with the principles of the Helsinki Declaration and was formally approved by the ethics committee of the Federal University of São Paulo (number: 0135/04).

\section{Anthropometric measurements and body composition}

Subjects were weighed while wearing light clothing and no shoes on a Filizola scale to the nearest $0.1 \mathrm{~kg}$. Height was measured to the nearest $0.5 \mathrm{~cm}$ with a wall-mounted stadiometer (Sanny, São Bernardo do Campo, SP, Brazil; model ES 2030). BMI was calculated as body weight divided by height squared (weight/height ${ }^{2}$ ). Body composition was measured by plethysmography in a BOD POD body composition system (version 1.69; Life Measurement Instruments, Concord, CA, USA).

\section{Measurements of visceral and subcutaneous fat}

All abdominal ultrasonography procedures and measurements of visceral and subcutaneous fat were performed by the same blinded diagnostic imaging specialist using a $3.5 \mathrm{MHz}$ multifrequency transducer (broadband). This procedure allowed a reduction in the risk margin for misclassification. The intra-examination coefficient of variation for ultrasonography was $0.8 \%$. Ultrasonography measurements of intra-abdominal (visceral) and subcutaneous fat were taken. Ultrasonography-determined subcutaneous fat was defined as the distance between the skin and external face of the rectus abdominis muscle, and visceral fat was defined as the distance between the internal face of the same muscle and the anterior wall of the aorta. ${ }^{18}$

\section{Measurements of carotid artery IMT}

Carotid artery IMT was measured using high-resolution ultrasound equipment (logic 5 and $\operatorname{logic} 7$, General Electric, São Paulo, SP, Brazil) with a $7-14 \mathrm{MHz}$ linear array transducer. Measurements were taken by the same experienced radiologist, who performed all scans and was unaware of the medical and biochemical status of the subject. The intra-examination coefficient of variation for IMT was $4.36 \%$. Patients were examined in the supine position with the neck in hyperextension. The protocol involved repeated measurements of the right and left common carotid far wall at $2 \mathrm{~cm}$ proximal to the bulb bifurcation. On a longitudinal B-mode image, the far wall of the common carotid artery appears as two bright, parallel lines separated by a hypoechoic space. The distance between the leading edge of the first bright line on the far wall (lumen-intima interface) and the leading edge of the second bright line (media-adventitia interface) indicates the IMT of the far wall, as previously described. ${ }^{19}$ Three measurements of the left and right common carotid were taken, and the mean for the higher measures of each side, representing the IMT, was used ${ }^{11}$ for statistical analysis.

\section{Serum analysis}

Blood samples were collected at the outpatient clinic at $\sim 0800$ hours after an overnight fast. After collection, the blood was centrifuged for $10 \mathrm{~min}$ at 5000 r.p.m. and stored at $-70^{\circ} \mathrm{C}$ for future analyses. The materials used for collection were disposable, adequately labeled and of recognized quality. Blood was collected by a skilled and qualified technician.

Insulin resistance can be defined as decreased sensitivity or responsiveness to the metabolic actions of insulin. ${ }^{10}$ The homeostasis model assessment of insulin resistance index (HOMA-IR) seems to be the index with the highest sensitivity and specificity for measuring insulin sensitivity and was the one selected for use in this study. The cutoff point adopted for adolescents was 3.16. ${ }^{20}$ HOMA-IR was calculated as the product of blood glucose (fasting blood glucose) and immunoreactive insulin (I): (fasting blood glucose (mg per $100 \mathrm{ml}) \times \mathrm{I}$ $\left.\left(\mathrm{mUl}^{-1}\right) / 405\right)$. The coefficient of variation for the insulin test was $3.3 \%$.

Dyslipidemia is a lipid metabolic alteration arising from disturbances in any lipid metabolism phase that alter serum concentrations of lipoprotein. To identify any individuals with abnormal lipid profiles, we used the reference values from the I Guideline for Preventing Atherosclerosis in Childhood and Adolescence. ${ }^{21}$ All variables were analyzed using a commercial kit (CELM, Barueri, Brazil).

The levels of adiponectin and PAI-1 were measured using a commercially available enzyme-linked immunosorbent assay kit from R\&D Systems (Minneapolis, MN, USA), according to the instructions of the manufacturer. The coefficients of variation for the adiponectin and PAI-1 procedure were 5.18 and $4.5 \%$, respectively.

\section{Blood pressure (BP)}

BP was measured on the right arm using a mercury-gravity manometer with proper cuff size, with the volunteers in the seated position. The first appearance of sound (phase 1 Korotkoff sound) was used to define systolic BP, and the disappearance of sound (phase 5 Korotkoff sound) was used to define diastolic BP. ${ }^{1}$ Hypertension was determined by reference values of BP percentiles for gender, according to age and height percentile. ${ }^{21}$

\section{Research design}

The interdisciplinary weight-loss program combined exercise training (aerobic plus resistance training) with clinical, nutritional and psychological therapy. The use of this kind of therapy has been suggested by the World Health Organization. ${ }^{22}$ All measurements were performed before and after 1 year of intervention.

Exercise protocol. The combined exercise training program was conducted three times per week for 1 year, and included $30 \mathrm{~min}$ of aerobic training plus $30 \mathrm{~min}$ of resistance training per session. The subjects were instructed to reverse the order of the exercises (aerobic and resistance) at each training session. The aerobic training consisted of running on a motor-driven treadmill (Life Fitness, Schiller Park, IL, USA, Model TR 9700HR) at a cardiac frequency intensity representing ventilatory threshold $\mathrm{I}$ ( \pm 4 b.p.m.), as determined by the results of an initial oxygen uptake test for aerobic exercises. The exercise program was based on guidelines from the American College of Sports Medicine. ${ }^{23}$

Resistance training was also designed based on American College of Sports Medicine recommendations. ${ }^{24}$ Exercises targeted each of the main muscle groups. After an introductory period, the load of training was adjusted such 
that volume and intensity were adjusted inversely, decreasing the number of repetitions to between 6 and 20 repetitions, for three sets.

All sessions were supervised individually by an experienced physiologist.

Psychological therapy. Psychological therapy treatment plans were established on the basis of validated questionnaires that considered some of the psychological problems caused by obesity, as described in the literature. These include depression, eating disorders, anxiety, decreased self-esteem and body image disorders. Interdisciplinary therapy consisted of a weekly 1-h group session. Individualized psychological therapy was recommended when weight problems were found.

Nutritional therapy. Energy intake was set at levels recommended for subjects with low levels of physical activity of the same age and gender following a balanced diet. Once a week, adolescents received nutrition lessons covering the topics related to a healthy eating pattern. All patients received individual nutritional consultation during the intervention program. At the beginning of the study and at 12 months into the program, a 3-day dietary record was collected. Because most obese people under report their food consumption, each adolescent was asked to record their diet with help from his or her parents. The degree of under reporting may still be substantial; however, this is a validated method for the assessment of dietary consumption. ${ }^{25}$ These dietary data were transferred to a computer by the same dietician, and the nutrient composition was analyzed by a PC program developed at the Federal University of São Paulo-Paulista Medicine School (Nutwin software, for Windows, version $1.5,2002$ ).

Clinical therapy. To address the health and clinical parameters, obese adolescents visited the endocrinologist once each month. Medical follow-up and treatment were based on an initial patient and family history, physical examination and intervention in any health problems that had developed over the course of the therapy.

\section{Statistical analysis}

Statistical analyses were performed using STATISTICA (Tulsa, OK, USA; version 7.0 for Windows). The Gaussian distribution of variables (including $\Delta$ values) was verified with a Shapiro-Wilk's $W$-test. Variables with normal distribution were expressed as mean \pm s.d., whereas variables without normal distribution were expressed as median (quartile range) in a descriptive table.
Non-parametric methods were used when appropriate (for BMI, visceral and subcutaneous fat, triglyceride levels, systolic and diastolic BP, carotid IMT, PAI1 and adiponectin concentration). Comparisons between measurements before and after intervention were performed with paired Student's $t$-tests for parametric variables and Wilcoxon's signed rank test for non-parametric variables. Pearson's correlation coefficients were calculated to assess possible relationships between normally distributed variables. Multiple regression analysis was used to verify the influence of changes in the traditional cardiovascular risk factors and adipokine concentrations on carotid IMT changes.

\section{RESULTS}

The clinical characteristics of the 29 subjects who completed the therapy with participation in $>75 \%$ of treatment sessions are shown in Table 1. The results are presented for the whole population studied, because we did not find significant gender differences in carotid IMT. Significant reductions were observed in total body mass, BMI, fat body mass ( $\%$ and $\mathrm{kg}$ ), visceral and subcutaneous fat, systolic and diastolic BP, insulin concentration, HOMA-IR, total cholesterol and low-density lipoprotein-cholesterol. In addition, 1 year of interdisciplinary intervention promotes a significant increase in lean body mass (\% and $\mathrm{kg}$ ) and high-density lipoprotein-cholesterol. The PAI-1 concentration and common carotid IMT were significantly reduced $(\Delta=-3.90 ; P \leqslant 0.01 ; \Delta=-0.06, P \leqslant 0.01$, respectively), whereas adiponectin concentration was increased $(\Delta=2.12, P \leqslant 0.01$; Figure 1$)$. According to nutritional profile data, the obese adolescents had significantly reduced their total energy intake, macronutrients (in grams) and lipid percentage by the end of the intervention (Table 2).

After analyzing the correlation between $\triangle$ HOMA-IR with lipid profile ( $\Delta$ triglycerides, $\Delta$ high-density lipoprotein-cholesterol, $\Delta$ low-density lipoprotein-cholesterol and $\Delta$ total cholesterol), inflammatory factors ( $\Delta \mathrm{PAI}-1$ and $\Delta$ adiponectin), $\Delta$ systolic BP and $\Delta$ diastolic BP, a negative correlation was found only between $\triangle$ HOMA-IR and changes in adiponectin concentration ( $\Delta$ adiponectin; $r=-0.42, P=0.02$; Figure 2a). Meanwhile, a positive correlation was observed between $\triangle$ HOMA-IR and changes in carotid IMT ( $\triangle \mathrm{IMT} ;=0.41, P=0.03$; Figure $2 \mathrm{~b}$ ).

Table 1 Clinical characteristic at baseline and after interdisciplinary intervention

\begin{tabular}{|c|c|c|c|c|}
\hline & Baseline $\mathrm{n}=29$ (17 female; 12 male $)$ & After 1 year $\mathrm{n}=29$ ( 17 female; 12 male $)$ & $\Delta$ & P-value \\
\hline Age & $16.03 \pm 1.59$ & - & & \\
\hline Total body mass (kg) & $102.31 \pm 13.35$ & $88.96 \pm 11.24$ & $-13.35 \pm 6.95$ & $\leqslant 0.01$ \\
\hline $\mathrm{BMI}\left(\mathrm{kg} \mathrm{m}^{-2}\right)$ & $35.19(33.13-38.85)$ & $31.19(27.78-33.45)$ & $-5.17 \pm 2.45$ & $\leqslant 0.01$ \\
\hline Fat body mass (\%) & $46.10 \pm 5.79$ & $35.94 \pm 7.74$ & $-10.16 \pm 4.44$ & $\leqslant 0.01$ \\
\hline Lean body mass (\%) & $53.90 \pm 5.79$ & $64.06 \pm 7.74$ & $10.16 \pm 4.44$ & $\leqslant 0.01$ \\
\hline Fat body mass (kg) & $47.52 \pm 10.44$ & $32.42 \pm 9.80$ & $-15.11 \pm 6.09$ & $\leqslant 0.01$ \\
\hline Lean body mass (kg) & $54.79 \pm 6.59$ & $56.56 \pm 6.87$ & $1.78 \pm 3.15$ & $\leqslant 0.01$ \\
\hline Waist circumference $(\mathrm{cm})$ & $103.58 \pm 9.79$ & $89.84 \pm 10.23$ & $-16.84 \pm 18.65$ & $\leqslant 0.01$ \\
\hline Visceral fat $(\mathrm{cm})$ & $3.80(3.10-4.50)$ & $2.19(1.80-2.90)$ & $-1.59 \pm 0.99$ & $\leqslant 0.01$ \\
\hline Subcutaneous fat $(\mathrm{cm})$ & $3.60(3.40-4.30)$ & $3.20(2.60-3.50)$ & $-1.00 \pm 0.82$ & $\leqslant 0.01$ \\
\hline $\mathrm{SBP}(\mathrm{mm} \mathrm{Hg})$ & $120(110-130)$ & $110(110-120)$ & $-9.66 \pm 16.74$ & $\leqslant 0.01$ \\
\hline $\mathrm{DBP}(\mathrm{mm} \mathrm{Hg})$ & $80(75-85)$ & $70(70-80)$ & $-6.14 \pm 7.13$ & $\leqslant 0.01$ \\
\hline Total cholesterol (mg per $100 \mathrm{ml}$ ) & $172.17 \pm 33.30$ & $155.38 \pm 30.81$ & $-16.79 \pm 21.69$ & $\leqslant 0.01$ \\
\hline LDL-c (mg per $100 \mathrm{ml})$ & $108.90 \pm 29.29$ & $94.55 \pm 25.81$ & $-14.34 \pm 17.18$ & $\leqslant 0.01$ \\
\hline HDL-c(mg per $100 \mathrm{ml})$ & $42.69 \pm 7.12$ & $45.00 \pm 8.02$ & $2.31 \pm 5.88$ & $\leqslant 0.01$ \\
\hline Triglycerides (mg per $100 \mathrm{ml}$ ) & $102.00(68.00-112.00)$ & $73.00(62.00-79.00)$ & $-23.97 \pm 37.75$ & $\leqslant 0.01$ \\
\hline Glucose (mg per $100 \mathrm{ml}$ ) & $88.97 \pm 7.02$ & $87.72 \pm 7.76$ & $-1.24 \pm 10.63$ & 0.85 \\
\hline Insulin $\left(\mu \cup \mathrm{ml}^{-1}\right)$ & $16.18 \pm 4.61$ & $8.93 \pm 3.71$ & $-7.24 \pm 4.34$ & $\leqslant 0.01$ \\
\hline HOMA-IR & $3.57 \pm 1.13$ & $1.97 \pm 0.92$ & $-1.69 \pm 1.21$ & $\leqslant 0.01$ \\
\hline
\end{tabular}

Abbreviations: BMI, body mass index; DBP, diastolic blood pressure; HDL-c, high-density lipoprotein-cholesterol; HOMA-IR, homeostasis model assessment insulin resistance index; LDL-c, lowdensity lipoprotein-cholesterol; SBP, systolic blood pressure.

Data expressed as mean \pm s.d. or median (quartile range).

Reference values ${ }^{20}$ : total cholesterol $<150$; $L D L-C<100 ; \mathrm{HDL}-\mathrm{c} \geqslant 45$; triglycerides $<100$. 
a

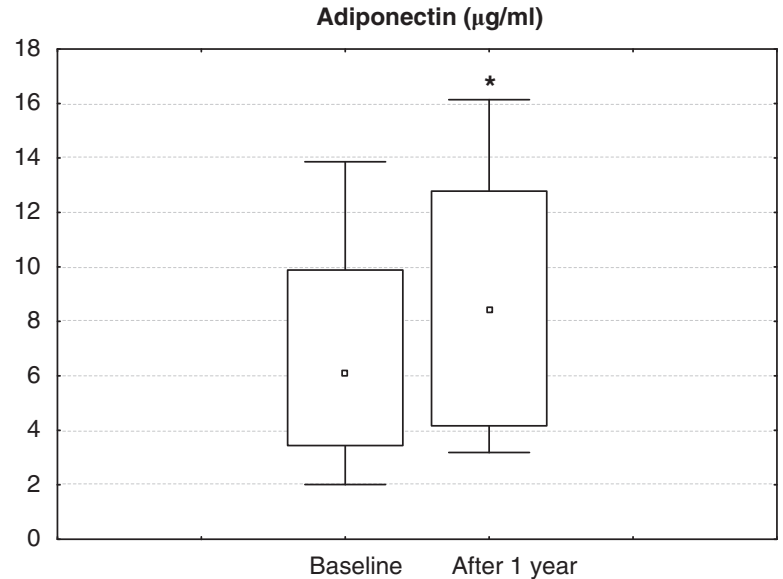

b

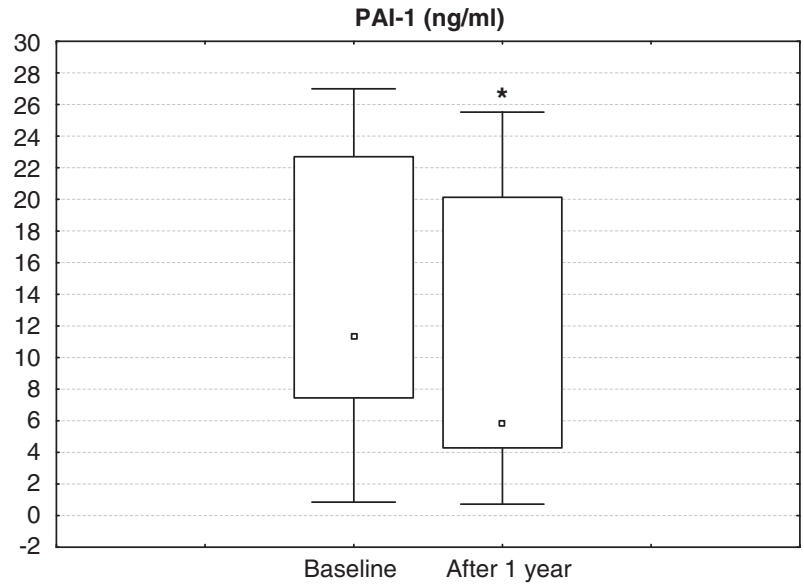

C

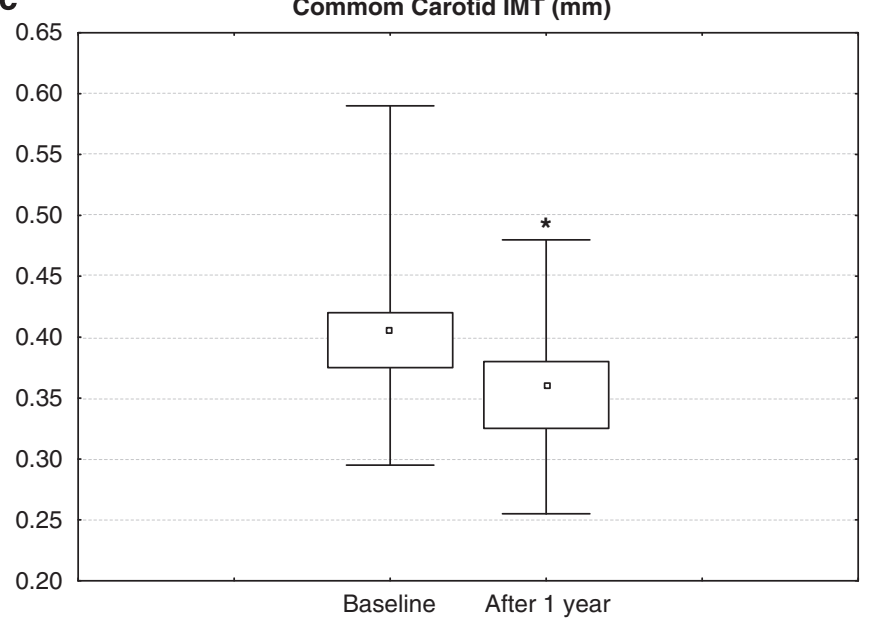

Figure 1 Values of Adiponectin concentration (a), PAI-1 concentration (b) and common carotid IMT (c) before and after interdisciplinary intervention $\left(n=29 ;{ }^{*} P \leqslant 0.01\right)$.

Multiple regression analysis adjusted by age, traditional cardiovascular risk factors and inflammatory markers revealed $\triangle$ HOMA-IR to be an independent predictor of significant reduction in carotid IMT ( $\beta=0.03, P=0.02$; Table 3).

It is also extremely important to emphasize that this interdisciplinary intervention reduced the prevalence of insulin resistance (from 66 to $17 \%$ ), dyslipidemia (from 17 to $10 \%$ ) and hypertension (from 41 to $0 \%$ ).
Table 2 Energy and macronutrients intake at baseline and after interdisciplinary intervention

\begin{tabular}{lcc}
\hline Nutrients & Baseline & After 1 year \\
\hline Energy intake (kcal) & $1900.85 \pm 586$ & $1331.25 \pm 444.42^{\#}$ \\
Carbohydrate (g) & $232.80 \pm 87.57$ & $177.90 \pm 51.62^{\#}$ \\
Carbohydrate (\%) & $50.50 \pm 6.72$ & $56.90 \pm 14.61$ \\
Protein (g) & $89.38 \pm 24.65$ & $63.54 \pm 24.35^{\#}$ \\
Protein (\%) & $19.27 \pm 4.16$ & $19.29 \pm 4.69$ \\
Lipid (g) & $66.59 \pm 27.26$ & $39.09 \pm 19.36^{\#}$ \\
Lipid (\%) & $31.03 \pm 4.96$ & $25.69 \pm 7.28^{\#}$ \\
\hline$P \leqslant 0.05$. & &
\end{tabular}

\section{DISCUSSION}

Considerable effort has been spent understanding the biology underlying the development of cardiovascular disease and identifying its risk factors. ${ }^{26}$ Several subclinical pathological conditions may be present during adolescence and can evolve over time, suggesting that further investigations will be required to investigate the primary prevention and early diagnosis of chronic diseases during this period. ${ }^{3}$

During recent years, our group has been dedicated to developing interdisciplinary interventions that aim to control obesity and its comorbidities, such as non-alcoholic fatty liver disease, ${ }^{25}$ metabolic syndrome ${ }^{1}$ and, now, atherosclerosis.

Recent research showed that $1 \mathrm{~h}$ of isolated aerobic training three times a week for 6 months was able to promote a reduction in body weight, BP, insulin resistance and carotid IMT in obese adolescents $\left(\mathrm{BMI}=29.8 \mathrm{~kg} \mathrm{~m}^{-2} \pm 5.9\right.$; age between 11 and 16 years). ${ }^{27}$ Another study demonstrated that a 6 month regimen of diet alone or diet plus exercise training decreased carotid IMT in overweight children (BMI $=25 \mathrm{~kg} \mathrm{~m}^{-2} \pm 3$; age between 9 and 12 years). ${ }^{14}$ In this study, 1 year of intervention promoted a significant decrease in carotid IMT in obese adolescents $\left(\mathrm{BMI}=36.50 \mathrm{~kg} \mathrm{~m}^{-2} \pm 4.99\right.$; age between 14 and 19 years). The most relevant guidelines in the field of cardiovascular disease prevention strongly emphasize the need for overweight and obese individuals to change their lifestyles in order to reduce body weight and thereby improve $\mathrm{BP}$ and lower cardiovascular disease risk. $^{28}$

To better explore the factors that precipitated the carotid IMT reduction in this study, multiple regression analysis was performed. It was found that change in HOMA-IR was an independent predictor of improvement in carotid IMT. A literature review by Reaven supports this hypothesis, strongly suggesting that insulin resistance is a physiological state associated with an increased risk of developing a cluster of abnormalities and clinical symptoms commonly observed in cardiometabolic syndrome. ${ }^{29}$

The influence of insulin resistance on the vasculature can occur directly and indirectly. Because insulin is a major stimulus for nitric oxide-mediated vasodilation, insulin resistance could contribute to impaired nitric oxide-mediated vasodilation, leading to endothelial dysfunction. Furthermore, insulin resistance also contributes to impaired lipid metabolism and activation of inflammatory signaling in the vasculature. However, the indirect mechanism may require a longer time to manifest, ${ }^{30}$ which could explain the lack of association between the changes in HOMA-IR and changes in lipid profiles and PAI-1, in this study.

Perhaps the most crucial idea suggested by this investigation is that the indirect influence of individual metabolic syndrome parameters on the risk of cardiovascular disease, including carotid IMT, may be 

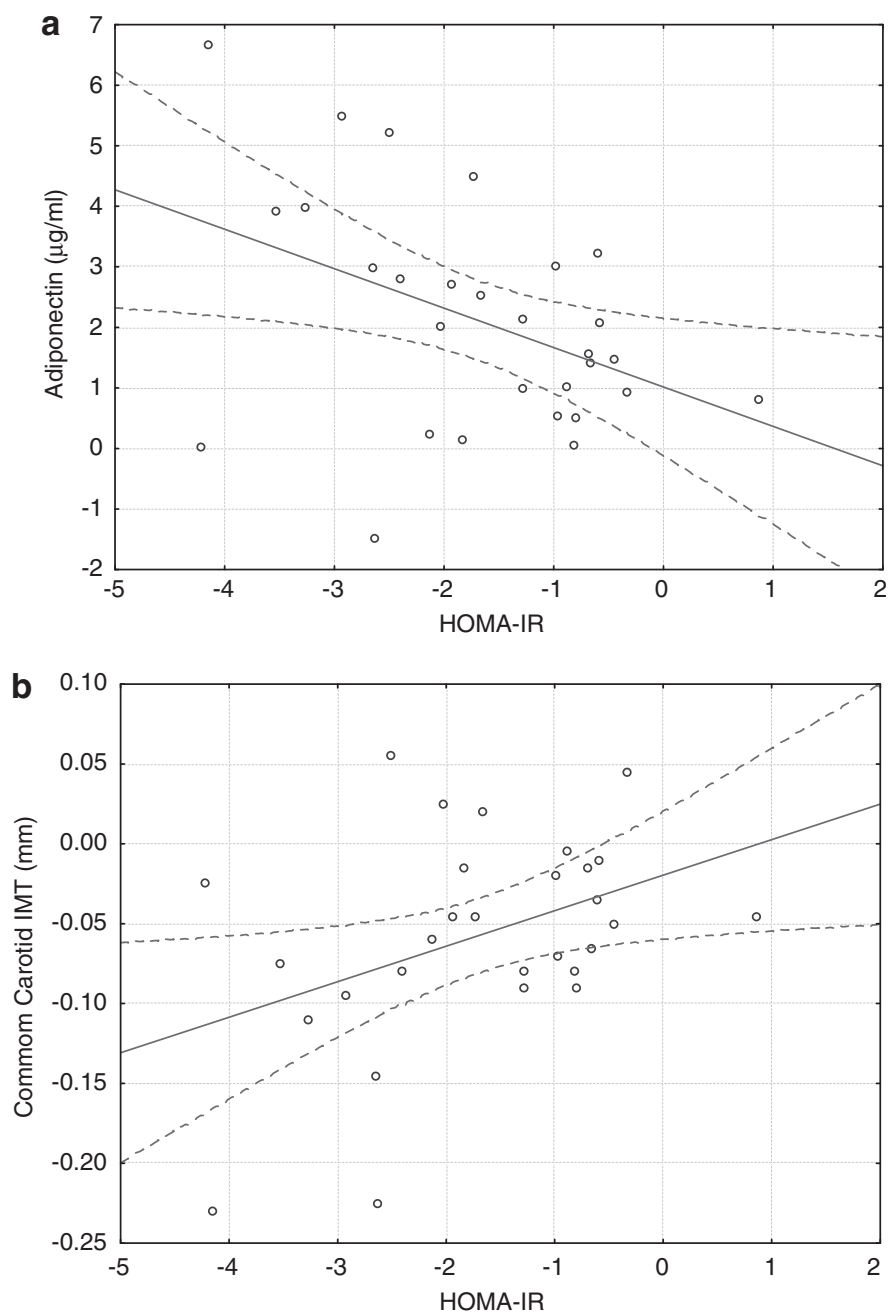

Figure 2 Correlations of $\triangle$ HOMA-IR with $\Delta$ adiponectin (a) and $\Delta$ common carotid IMT (b) in obese adolescents. (a) $n=29 ; r=-0.42 ; P=0.02$. (b) $n=29$; $r=0.41 ; P=0.03$.

Table 3 Multiple regression analysis for the determinants of changes in common carotid IMT

\begin{tabular}{lcc}
\hline & \multicolumn{2}{c}{$\Delta$ Common carotid IMT } \\
\cline { 2 - 3 } & Regression coefficient $\beta$ & $\mathrm{P}$ \\
\hline Age & 0.01 & 0.19 \\
$\Delta$ LDL-c (mg per 100 ml) & -0.00 & 0.62 \\
$\Delta$ SBP $(\mathrm{mm} \mathrm{Hg})$ & -0.00 & 0.69 \\
$\Delta \mathrm{DBP}(\mathrm{mm} \mathrm{Hg})$ & -0.00 & 0.50 \\
$\Delta$ Visceral fat $(\mathrm{cm})$ & 0.02 & 0.22 \\
$\Delta$ HOMA-IR & 0.03 & 0.02 \\
$\Delta$ PAl-1 $\left(\mathrm{ng} \mathrm{ml}^{-1}\right)$ & 0.00 & 1.00 \\
\hline
\end{tabular}

Abbreviations: DBP, diastolic blood pressure; HOMA-IR, homeostasis model assessment insulin resistance index; IMT, intima-media thickness; LDL-c, low-density lipoprotein-cholesterol; PAI-1, plasminogen activator inhibitor type-1; SBP, systolic blood pressure.

mediated through insulin resistance. However, this should be confirmed in a large cohort study. This finding would be not only biologically important but also clinically significant, as it would allow insulin resistance to become the main focus in the prevention of atherosclerosis in obese adolescents.
When we analyzed the nutritional profile of the study population, it was observed that 1 year of interdisciplinary therapy promoted a significant reduction in total energy intake, macronutrients (in grams) and lipid percentage, reinforcing the importance of nutritional counseling in obesity treatment as well as in cardiovascular disease prevention. This finding is in line with results reported by Semenkovich, ${ }^{31}$ which suggested that the delivery of excess fuel may cause mitochondrial stress, which can lead to increased production of reactive oxygen species, further contributing to endothelial dysfunction and the inflammatory state. Therefore, this study recommends reducing fat intake and increasing physical activity as strategies to minimize the impact of obesity on inflammatory disorders. ${ }^{27} \mathrm{~A}$ similar interdisciplinary intervention should be considered as a potential therapeutic strategy.

Our intervention was also able to promote a significant increase in serum adiponectin concentration $(P \leqslant 0.01)$, which probably contributed to the improvement in insulin resistance and decrease in carotid IMT. Previous studies have demonstrated that this adipokine stimulates potent anti-inflammatory and atheroprotective responses in vascular tissue, in addition to its insulin-sensitizing effects in tissues involved in glucose and lipid metabolism., ${ }^{7,9}$ 
Thus, the reduced circulating concentration of adiponectin observed in obese subjects could contribute not only to insulin resistance but also to endothelial vascular dysfunction. Adiponectin may be more closely related to atherosclerosis in obese children than are conventional cardiovascular risk factors and inflammation status. ${ }^{6}$ For example, it has been demonstrated that high adiponectin concentration can suppress the development of atherosclerosis in susceptible mice. ${ }^{32}$ Consistent with this hypothesis, our study verified a negative correlation between change in HOMA-IR and change in adiponectin concentration.

Additionally, insulin resistance and inflammation may promote an altered profile of vascular function and, consequently, hypertension. Insulin resistance increases the endothelial expression of PAI-1 and cellular adhesion molecules, ${ }^{33,34}$ suggesting that multiple mechanisms may contribute to accelerated atherosclerosis. ${ }^{7,35}$ Thus, the decrease in PAI-1 concentration observed in this investigation may help to attenuate the monocyte migratory capacity mediated by insulin in a pro-inflammatory and atherothrombotic state. This mechanism should be investigated more fully.

The small sample size used in this study can be considered as a limitation of our study. Nevertheless, for the first time, we showed that a change in HOMA-IR was an independent predictor for change in carotid IMT among obese Brazilian adolescents. Another finding of great value in this investigation was a negative correlation between change in HOMA-IR and changes in adiponectin concentration. Beauloye et al. ${ }^{6}$ showed a negative correlation between adiponectin and carotid IMT in children and adolescents, but only at baseline conditions. These results reinforce the importance of adopting strategies that promote an improvement of HOMA-IR and adiponectinemia, which could influence IMT measurements.

It is of utmost relevance that the IMT change is multifactorial ${ }^{36}$ and, in this age group, may be strongly influenced by fatty acid and inflammatory infiltration, despite the improvement in other variables considered to cause the reduction in carotid artery IMT.

Although some of our patients remained obese at the end of therapy, the changes in lifestyle (mostly increased physical activity and decreased food intake) promoted by this type of intervention can be considered essential to create a better metabolic profile and thereby reduce atherosclerotic risk. Moreover, these results showed that these patients require intensive and long-term therapy. Furthermore, study recruitment and compliance with any treatment is more difficult in adolescent populations, and the success of treatment depends at least partly on the participation of parents and/or guardians. ${ }^{37}$

In conclusion, this shows that obesity intervention may prevent the development of cardiovascular disease among adolescents.

\section{ACKNOWLEDGEMENTS}

We thank the patients who participated in this study and the following funding sources: AFIP, FAPESP 2006/00684-3, FAPESP 2008/53069-0, FAPESP (CEPID/ Sleep \#9814303-3 S.T) CNPq, CAPES, CENESP, FADA and UNIFESP-EPM, which supported the CEPE-GEO Interdisciplinary Obesity Intervention Program. We also express our gratitude to Leandro Stetner for his assistance with statistical analysis and data presentation.

1 Caranti DA, Lazzer S, Dâmaso AR, Agosti F, Zennaro R, de Mello MT, Tufik S, Sartorio A. Prevalence and risk factors of metabolic syndrome in Brazilian and Italian obese adolescents: a comparison study. Int J Clin Pract 2008; 10: 1526-1532.

2 Oda E. The metabolic syndrome as a concept of adipose tissue disease. Hypertens Res 2008; 31: 1283-1291.
3 Rodrigues AN, Perez AJ, Pires JG, Carletti L, Araújo MT, Moyses MR, Bissoli NS, Abreu GR. Cardiovascular risk factors, their associations and presence of metabolic syndrome in adolescents. J Pediatr 2009; 85: 55-60.

4 Järvisalo MJ, Jartti L, Näntö-Salonen K, Irjala K, Rönnemaa T, Hartiala JJ, Celermajer DS, Raitakari OT. Increased aortic intima-media thickness: a marker of preclinical atherosclerosis in high-risk children. Circulation 2001; 104: 2943-2947.

5 Reinehr T, Kiess W, de Sousa G, Stoffel-Wagner B, Wunsch R. Intima-media thickness in childhood obesity. Relations to inflammatory marker, glucose metabolism, and blood pressure. Metab Clin Exp 2006; 55: 113-118.

6 Beauloye V, Zech F, Tran HT, Clapuyt P, Maes M, Brichard SM. Determinants of early atherosclerosis in obese children and adolescents. J Clin Endocrinol Metab 2007; 92: 3025-3032.

7 Shargorodsky M, Boaz M, Goldberg Y, Matas Z, Gavish D, Fux A, Wolfson N. Adiponectin and vascular properties in obese patients: is it a novel biomarker of early atherosclerosis? Int J Obesity 2009; 33: 553-558.

8 Bruun JM, Lihn AS, Verdich C, Pedersen SB, Toubro S, Astrup A, Richelsen B. Regulation of adiponectin by adipose tissue-derived cytokines: in vivo and in vitro investigations in humans. Am J Physiol Endocrinol Metab 2003; 285: E527-E533.

9 Goldstein BJ, Scalia R. Adiponectin: a novel adipokine linking adipocytes and vascular function. J Clin Endocrinol Metab 2004; 89: 2563-2568.

10 Muniyappa R, Lantorno M, Quon MJ. An integrated view of insulin resistance and endothelial dysfunction. Endocrinol Metab Clin N Am 2008; 37: 685-711.

11 Rodriguez RR, Gómez-Díaz RA, Haj JT, Garnica FJA, Soriano ER, Meguro EN, AguilarSalinas CA, Wacher NH. Carotid intima-media thickness in pediatric Type 1 diabetic patients. Diabetes Care 2007; 30: 2599-2602.

12 Kim K, Valentine RJ, Shin Y, Gong K. Associations of visceral adiposity and exercise participation with C-reactive protein, insulin resistance, and endothelial dysfunction in Korean health adults. Metabolism 2008; 57: 1181-1189.

13 Hadi HAR, Car CS, Suwaidi JA. Endothelial dysfunction: cardiovascular risk factors, therapy and outcome. Vasc Health Risk Managem 2005; 1: 183-198.

14 Woo KS, Chook P, Yu CW, Sung RYT, Qiao M, Leung SSF, Lam CWF, Metreweli C, Celermajer DS. Effect of diet and exercise on obesity related vascular dysfunction in children. Circulation 2004; 109: 1981-1986.

15 Wunsch R, de Sousa G, Toschke AM, Reinehr T. Intima-media thickness in obese children before and after weight loss. Pediatr 2006; 118: 2334-2340.

16 Tanner JM, Whitehouse RH. Clinical Longitudinal standards for height, weight, weight velocity and stages of puberty. Arch Dis Child 1976; 51: 170-179.

17 Centers for Disease Control and Prevention. Hyattsville: National Center for Health Statistics. (Updates on 11 January 2007; accessed on 15 August 2007). Prevalence of overweight among children and adolescents: United States 1999-2002. http:// www.cdc.gov/nchs/products/pubs/pubd/hestats/overwght99.htm.

18 Ribeiro-Filho FF, Faria NA, Azjen S, Zanella MT, Ferreira SR. Methods of estimation of visceral fat: advantages of ultrasonography. Obes Res 2003; 11: 1488-1494.

19 Guardamagna O, Abello F, Saracco P, Baracco V, Rolfo E, Pirro M. Endothelial activation, inflammation and premature atherosclerosis in children with familial dyslipidemia. Atherosclerosis 2009; 207: 471-475.

20 Keskin M, Kurtoglu S, Kendirci M, Atabek ME, Yazici C. Homeostasis model assessment is more reliable than the fasting glucose/insulin ratio and quantitative insulin sensitivity check index for assessing insulin resistance among obese children and adolescents. Pediatrics 2005; 115: e500-e503.

21 Giuliano ICB, Caramelli B, Pellanda L, Duncan B, Mattos S, Fonseca FAH. I Guideline for preventing atherosclerosis in childhood and adolescence. Int J Atheroscler 2006; 1: $1-30$.

22 World Health Organization (WHO). Report of a WHO consultation. In: Alwan A, King H (eds). Definition, Diagnosis and Classification of Diabetes Mellitus and its Complications. Part 1: Diagnosis and Classification of Diabetes Mellitus. Department of Noncommunicable Disease Surveillance, World Healthy Organization: Geneva, 1999, pp 1-59.

23 Donnelly JE, Blair SN, Jakicic JM, Manore MM, Rankin JW, Smith BK. Appropriate physical activity intervention strategies for weight loss and prevention of weight regain for adults. Med Sci Spor Exerc 2009; 41: 459-471.

24 American College of Sports Medicine. Progression models in resistance training for healthy adults. Med Sci Spor Exerc 2002; 34: 364-380.

25 de Piano A, Prado WL, Caranti DA, Siqueira KO, Stella SG, Lofrano M, Tock L, Cristofalo DM, Lederman H, Tufik S, de Mello MT, Dâmaso AR. Metabolic and nutritional profile of obese adolescents with nonalcoholic fatty liver disease. J Pediatr Gastroenterol Nutr 2007; 44: 446-452.

26 Velasquez-Mieyer P, Neira CP, Nieto R, Cowan PA. Obesity and cardiometabolic syndrome in children. Ther Adv Cardiovasc Dis 2007; 1: 61-82.

27 Meyer AA, Kundt G, Lenschow U, Schuff-Werner P, Kienast W. Improvement of early vascular changes and cardiovascular risk factors in obese children after a six-month exercise program. J Am Coll Cardiol 2006; 48: 1865-1870.

28 Cícero AFG. Weight loss and blood pressure normalization: the relevance of early intervention in hypertension. Hypertens Res 2010; 33: 195-196.

29 Reaven GM. The metabolic syndrome: requiescat in pace. Clin Chem 2005; 51 : 931-938.

30 Razani B, Chakravarthy MV, Semenkovich CF. Insulin resistance and atherosclerosis. Endocrinol Metab Clin N Am 2008; 37: 603-621.

31 Semenkovich CF. Insulin resistance and atherosclerosis. J Clin Invest 2006; 116: 1813-1822.

32 Okamoto Y, Kihara S, Ouchi N, Nishida M, Arita Y, Kumada M, Ohashi K, Sakai N, Shimomura I, Kobayashi H, Terasaka N, Inaba T, Funahashi T, Matsuzawa Y. 
Adiponectin reduces atherosclerosis in apolipoprotein E-deficient mice. Circulation 2002; 106: 2767-2770.

33 Reaven GM. Insulin resistance: the link between obesity and cardiovascular disease. Endocrinol Metab Clin N Am 2008; 37: 581-601.

34 Maher V, O'Dowd M, Carey M, Markham C, Byrne A, Hand E, Mc Inerney D. Association of central obesity with early carotid intima-media thickening is independent of that from other risk factors. Int J Obesity 2009; 33: 136-143.
35 Kotsis V, Stabouli S, Papakatsika S, Rizos Z, Parati G. Mechanisms of obesity-induced hypertension. Hypertens Res 2010; 33: 386-393.

36 Kelishad R, Hashemi M, Mohammadifard N, Asgary S, Khavarian N. Association of changes in oxidative and proinflammatory states with changes in vascular function after a lifestyle modification trial among obese children. Clin Chem 2008; 54: 147-153.

37 Steinbeck K, Baur L, Cowell C, Pietrobelli A. Clinical research in adolescents: challenges and opportunities using obesity as a model. Int J Obesity 2009; 33: 2-7. 